\title{
Incidence and characterization of salmonella species in street food and clinical samples.
}

\begin{abstract}
The objectives of our study were to investigate the Salmonella species contamination in various types of ready-to-eat street-vended dishes or drinks, to isolate Salmonella spp. from clinical samples and to assess the possible relationship between the serotypes isolated from these two different environments. The isolates were characterized by their antibiotic resistance, plasmid profiles and randomly amplified polymorphic DNA (RAPD) sequences. A total of 24 salmonellae, belonging to seven different serotypes, were isolated from 129 different street-vended foods and drinks and 12 clinical samples (rectal swabs). The encountered serotypes from street foods were Salmonella Biafra $(n=8)$, Salmonella Braenderup $(\mathrm{n}=3)$ and Salmonella Weltevreden $(\mathrm{n}=1)$, and from clinical samples were Salmonella Typhi $(n=8)$, Salmonella Typhimurium $(n=2)$, Salmonella Paratyphi A $(n=1)$ and Salmonella Paratyphi B $(\mathrm{n}=1)$. The results showed no similarities in the types of Salmonella serotypes from street food and clinical samples examined. The Salmonella strains were resistant to one or more of the 14 tested antibiotics. Seventeen isolates harbored plasmids, with plasmid sizes ranging from 3.0 to 38.5 MDa. RAPD fingerprinting with primers OPAR3 and OPAR8 produced a combination of 21 fingerprint patterns. The dendrograms generated for the S. Biafra and S. Typhi showed strains of the same serotypes but of very different types of sample and location of sampling clustered together, indicating the possibility of cross contamination during food handling.
\end{abstract}

Keyword: Salmonella; Street food; Clinical samples. 